\title{
Der Bund und die FMH kommen auf das gleiche Resultat
}

\section{Esther Kraft}

Abteilungsleiterin DDQ

\author{
An der Erhebung "MAS 2015» (für Medical Ambulatory Structure) haben knapp \\ 7000 Arztpraxen und ambulante Zentren Angaben über ihre Tätigkeit, Umsatz und \\ Betriebsergebnis gemacht. Die Rücklaufquote betrug insgesamt gegen $75 \%$. Der Ab- \\ gleich der MAS-2015-Ergebnisse mit ärzteeigenen Daten zeigt vergleichbare Ergeb- \\ nisse trotz unterschiedlicher Datenquellen. Das durchschnittliche Ergebnis aus \\ Praxis- und anderen Tätigkeiten beträgt 155000 CHF, wobei 89\% der Praxistätigkeit \\ prämienfinanziert sind.
}

\section{Ausgangslage}

Am 20. März 2018 publizierte das Bundesamt für Statistik BFS die Analyse der Teilnahmezahlen und der Grundgesamtheit aller Ärzte für die erste Erhebung «Strukturdaten der Arztpraxen und ambulanten Zentren (MAS) [1]. Rund drei Viertel der angeschriebenen Arztpraxen und ambulanten Zentren lieferten Angaben zu ihrer Situation. Fast 7000 Arztpraxen und ambulante Zentren, und damit die Hälfte der zur bereinigten Grundgesamtheit gehörenden Unternehmen, haben den Fragebogen ausgefüllt und Daten geliefert [1]. Darauf basierend war es dem BFS weiter möglich, am 10. April 2018 die ersten Ergebnisse aus der Erhebung MAS 2015 zu publizieren [2]. Die Erhebung der Daten zu statistischen Zwecken für MAS 2015 erfolgte auf der Basis von Artikel 23 des Krankenversicherungsgesetzes KVG und in Anwendung des Bundesstatistikgesetzes (BStatG).

Tabelle 1: Anzahl Ärzte im praxisambulanten Sektor 2015 (Arztpraxen, Praxiszentren).

\begin{tabular}{|c|c|c|c|}
\hline & MAS 2015 & $\begin{array}{l}\text { FMH-Ärztestatistik } 2015 \\
\text { (ambulanter Sektor) }\end{array}$ & Delta \\
\hline $\begin{array}{l}\text { Berufstätige Ärztinnen und Ärzte } \\
\text { im praxisambulanten Sektor }\end{array}$ & 17599 & 18128 & $3,0 \%$ \\
\hline Vollzeitäquivalente & 14044 & 15046 & $7,1 \%$ \\
\hline $\begin{array}{l}\text { Arbeitspensum } \\
\text { (Halbtage pro Woche) }\end{array}$ & 7,6 & 8,3 & $9,2 \%$ \\
\hline $\begin{array}{l}\text { Pensum Männer } \\
\text { (Halbtage pro Woche) }\end{array}$ & 8,1 & 8,9 & $9,9 \%$ \\
\hline $\begin{array}{l}\text { Pensum Frauen } \\
\text { (Halbtage pro Woche) }\end{array}$ & 7,6 & 6,9 & $-9,2 \%$ \\
\hline
\end{tabular}

Somit kann die erste Erhebung als Erfolg betrachtet werden. Gemäss dem BFS war eine wichtige «Voraussetzung für die Teilnahme der Ärzteschaft an der Erhebung MAS 2015, dass die FMH als wichtigste Berufsorganisation sowie ihre Partner (Ärztekasse und NewIndex) die Datenlieferung zu statistischen Zwecken unterstützten. Schnittstellen zu den bestehenden Datensammlungen der Ärztekasse (RoKo), der NewIndex, der FMH und des MedReg vereinfachten den Unternehmen die Datenerfassung. Einerseits konnten dadurch mit wenigen Klicks bestehende Daten importiert, andererseits eine hohe Datenqualität gewährleistet werden» [1].

\section{Analyse der Daten durch die FMH}

Die Ergebnisse aus der Erhebung MAS 2015 sind für die FMH und die Ärzteschaft von zentraler Bedeutung und Interesse. Auf Grundlage des Bundesstatistikgesetzes und der Verordnung über die Durchführung von statistischen Erhebungen des Bundes sowie des Datenschutzgesetzes machte die FMH von der Möglichkeit Gebrauch, die Daten der Erhebung MAS 2015 mit einer klaren Zweckformulierung betreffend Forschung, Planung und Statistik beim BFS zu beantragen.

Der Abgleich der Ergebnisse aus der MAS-2015-Erhebung mit den ärzteeigenen Daten hinsichtlich Qualität, Aussagekraft und Interpretation soll eine transparente und faktenbasierte Diskussion auf Basis einer validierten Datengrundlage ermöglichen. 
Tabelle 2: Aufwand und Umsatz Einzelunternehmen MAS 2015 und RoKo.

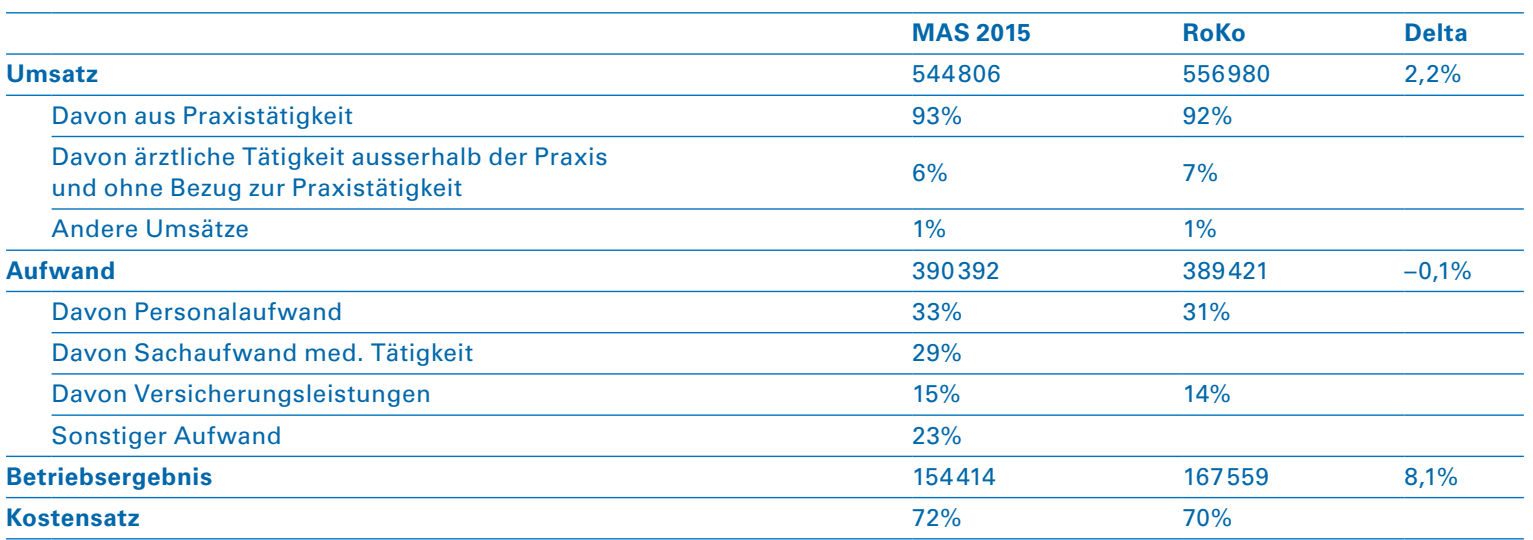

\section{Abgleich der Ärztezahlen und der Finanzdaten}

In einer ersten Analysephase wurden die Ergebnisse zur Anzahl berufstätiger Ärzte und deren Arbeitspensum aus der MAS-2015-Erhebung und der FMH-Ärztestatistik verglichen. In einem zweiten Schritt wurden die Finanzdaten aus der MAS-Erhebung den verfügbaren Daten aus der Rollenden Kostenstudie (RoKo) gegenübergestellt.

Das BFS weist in seiner Publikation für das Jahr 2015 17599 praxisambulant berufstätige Ärztinnen und Ärzte aus. Diese entsprechen 14044 Vollzeitäquivalenten. Die FMH-Ärztestatistik wies für das gleiche Jahr 18858 praxisambulant berufstätige Ärztinnen und Ärzte entsprechend 15046 Vollzeitäquivalenten aus (vgl. Tab. 1).

Bei den Finanzdaten für das Jahr 2015 weist das BFS für als Einzelunternehmen organisierte Arztpraxen einen Gesamtumsatz von durchschnittlich 545000 Franken aus. Der durchschnittliche Gesamtaufwand dieser Praxen lag bei 390000 Franken. Entsprechend ergibt dies einen Kostensatz (Aufwand/Umsatz) von durchschnittlich 72\%. Das Betriebsergebnis für die Einzelpraxen betrug im Jahr 2015 somit 155000 Franken. Im Vergleich dazu zeigen sich auf Basis der Daten der Rollenden Kostenstudie (RoKo) ein Kostensatz (Aufwand/ Umsatz) von $70 \%$ und ein Betriebsergebnis von rund 167000 Franken.

Korrespondenz: FMH Abteilung DDQ Elfenstrasse 18 CH-3000 Bern 15 Tel. 0313591111 ddq[at]fmh.ch
Bei den Daten der MAS-2015-Erhebung in Tabelle 2 ist zu beachten, dass die Ärztinnen und Ärzte bei den Einzelunternehmen nicht als Arbeitnehmende gelten. Entsprechend wird das Einkommen der Ärztinnen und Ärzte nicht unter dem Personalaufwand ausgewiesen.
Gemäss MAS-2015-Daten wird der Umsatz aus Praxistätigkeit (also 93\% des Gesamtumsatzes) zu 89\% aus der obligatorischen Krankenversicherung generiert. Bei einem durchschnittlichen Betriebsergebnis von 155000 Franken verdient der Praxisinhaber folglich 137950 Franken aus OKP-Prämiengeldern.

\section{Fazit}

Erfreulicherweise zeigen die unabhängigen Erhebungen des Bundes und der Ärzteschaft vergleichbare Ergebnisse betreffend die Anzahl Ärztinnen und Ärzte und deren Arbeitspensum sowie betreffend Finanzdaten. Die vorliegenden Daten bieten somit eine gute Grundlage für eine faktenbasierte und transparente Diskussion in Hinblick auf Ärztezahlen und die finanziellen Rahmenbedingungen der freipraktizierenden Ärzteschaft.

An dieser Stelle gilt unser Dank den Arztpraxen und praxisambulanten Zentren sowie allen Ärztinnen und Ärzten. Dank ihrer Transparenz und Datenbekanntgabe an das BFS sowie an die ärzteeignen Organisationen haben sie diese Ergebnisse erst ermöglicht.

\section{Literatur}

1 Erste Erhebung «Strukturdaten der Arztpraxen und ambulanten Zentren» (MAS 2015): Analyse von Teilnahme und Grundgesamtheit, Bundesamt für Statistik BFS, 20.3.2018; https://www.bfs. admin.ch/bfs/de/home/statistiken/gesundheit/erhebungen/sdapaz.assetdetail.4842232.html

2 Statistik der Arztpraxen und ambulanten Zentren (MAS), Bundesamt für Statistik BFS, 10.4.2018; https://www.bfs.admin.ch/bfs/de/ home/statistiken/gesundheit/gesundheitswesen/artzpraxen. assetdetail.4924760.html 